\title{
The role of phonological and visual working memory in complex arithmetic for Chinese- and Canadian-educated adults
}

\author{
INEKE IMBO \\ Ghent University, Ghent, Belgium \\ AND \\ JO-ANNE LEFEVRE \\ Carleton University, Ottawa, Ontario, Canada
}

\begin{abstract}
Two experiments were conducted to test cultural differences in the role of phonological and visual working memory in complex arithmetic. Canadian- and Chinese-educated students solved complex subtraction problems (e.g., 85 - 27; Experiment 1) and complex multiplication problems (e.g., $6 \times 13$; Experiment 2) under phonological and visual working memory loads. Problem complexity (i.e., borrow or carry operations) and presentation format (i.e., horizontal vs. vertical) were also manipulated. The results showed that both Chinese- and Canadian-educated participants relied on both phonological and visual working memory resources when solving complex subtraction and multiplication problems. Selective involvement of phonological and visual working memory as a function of operation (Lee \& Kang, 2002) or presentation format (Trbovich \& LeFevre, 2003) was found only for Chinese-educated participants and not for Canadian-educated participants, calling into question the generalizability of these findings across arithmetic operations and cultural groups.
\end{abstract}

Mental arithmetic is a skill that people use every day. Some arithmetic problems, such as $3+2$ or $8-4$, are relatively easy to solve. However, for more complex problems, such as $45+29$ or $78-15$, greater mental effort is required and a variety of mental processes are recruited. In the present research, we explored the role of phonological and visual working memory processes in solving complex subtraction and multiplication problems. Although it is generally acknowledged that working memory plays a significant role in arithmetic problem solving (DeStefano \& LeFevre, 2004), the involvement of working memory resources seems to depend on various factors, such as presentation format (horizontal vs. vertical), operation (subtraction vs. multiplication), and problem complexity (easy vs. difficult). One goal of the present study was to determine whether phonological and visual working memory resources were invoked differently depending on these factors.

In addition to the role of problem-based factors in arithmetic, the involvement of working memory may vary with educational experiences and arithmetic skill (Imbo \& LeFevre, 2009). Assessment of the working memory demands of specific operations or particular formats usually has been done only for relatively homogeneous groups, however, and thus generalizability of the results may be limited. To explore this issue, in the present research we tested participants from two cultures:
Chinese-educated Asians and Canadian-educated North Americans. In the domain of simple arithmetic, Asians are more skilled than North Americans (e.g., Campbell \& Xue, 2001; LeFevre \& Liu, 1997), and this advantage may exist even before children enter elementary school (Siegler \& Mu, 2008). Thus, our second goal in the present research was to evaluate the hypothesis that cultural differences in solution processes, and hence in working memory demands, may also be found in the domain of complex arithmetic.

The working memory model of Baddeley and Hitch (1974) and Baddeley (2000) has proven to be useful in research on working memory and mental arithmetic. According to this model, four components constitute working memory: The central executive is a modalityfree, limited-capacity system that includes control processes, monitoring, response selection, planning, and sequencing; the phonological loop and the visuospatial sketchpad store phonological and visuospatial information, respectively; and the episodic buffer combines temporary working memory information with long-term memory information.

In the present study, we investigated the involvement of two working memory components, the phonological loop and the visuospatial sketchpad, in two arithmetic operations: subtraction and multiplication. We explored

I. Imbo, ineke.imbo@ugent.be 
the extent to which previously obtained results regarding working memory and arithmetic are generalizable across groups differing in culture, arithmetic skill, and educational history. Our examination of cultural differences was, in part, motivated by the observation that the role of working memory components has rarely been examined for the same operations across cultures. For example, there is some support for a role of the phonological loop in subtraction and multiplication, but it is culture specific in that effects have been observed for Germans solving multiplication problems (Seitz \& Schumann-Hengsteler, 2000, 2002), for Americans solving subtraction problems (Seyler, Kirk, \& Ashcraft, 2003), and for Belgians solving multiplication and subtraction problems (Imbo, Vandierendonck, \& Vergauwe, 2007). There are no cross-cultural comparisons and no published accounts of Asian-educated adults solving complex arithmetic problems. Such comparisons would be of interest, because two possible roles for the phonological loop in multiplication have been proposed and these roles may vary across cultures as well as across problem complexity.

For simple multiplication, facts may be stored in an internal phonological code (Cohen \& Dehaene, 2000; Cohen, Dehaene, Chochon, Lehéricy, \& Naccache, 2000; Dehaene, 1992, 1997; Dehaene \& Cohen, 1995, 1997; Lee $\&$ Kang, 2002). Because Asian students practice multiplication using rote memorization and verbal repetition, the role of phonological codes may be greater for Asian students than for Canadian students (Campbell \& Xue, 2001; LeFevre \& Liu, 1997). Thus, we predicted that, for multiplication, Chinese-educated participants might need more phonological resources than Canadian-educated participants did.

In contrast, the role of the phonological loop in complex problems, both multiplication and subtraction, may be to store intermediate solutions (Ashcraft, 1995). For example, solving $56 \times 4$ involves the subproblems $4 \times 6$, $4 \times 50$, and $24+200$. The intermediate products, 24 and 200 , may be stored in the phonological loop. Similarly, for the problem $46-19$, the phonological loop may be used to maintain intermediate components of the solution, such as the borrowing of 10 from 40 and the unit solution, 7 , while the decade value is being processed. Thus, we predicted that both subtraction and multiplication would involve the phonological loop because of the possible storage function and that this would be true for both Chineseand Canadian-educated participants. Overall, therefore, the use of a phonological code in multiplication seems more likely for Chinese- than for Canadian-educated individuals, because the former rely more heavily on rote memorization of multiplication facts (e.g., Campbell \& Xue, 2001; LeFevre \& Liu, 1997), whereas both groups are likely to use the phonological loop for storage of intermediate solutions. Thus, cross-cultural comparisons will address the question of whether effects of working memory can be linked to differences in how arithmetic facts are stored and processed.

In contrast, there is less research supporting a role for visuospatial working memory in complex arithmetic; therefore, there are few specific predictions. Lee and Kang (2002) found that a visuospatial task interfered with performance on simple subtraction, but not on multiplication problems. For simple subtraction (and possibly addition), some researchers have proposed that activation of an internal magnitude code or number line representation occurs (e.g., Dehaene, 1992, 1997; Dehaene \& Cohen, 1995, 1997) and thus engages visual or spatial codes in working memory. Little support for this proposal has been found for simple addition or subtraction, but no researchers other than Lee and Kang have evaluated the hypothesis within a working memory framework.

For complex arithmetic, only Trbovich and LeFevre (2003) have observed significant visual load effects. In their study, Canadian-educated participants solved horizontally or vertically presented one-digit + two-digit addition problems (e.g., $8+43$ ) under phonological and visual working memory loads. Trbovich and LeFevre found decrements in performance with both types of load, but the phonological load affected horizontally presented problems more, whereas a visual load affected vertically presented problems more. These results were interpreted in the light of the participants' strategy choices. Trbovich and LeFevre hypothesized that vertically presented problems are likely to activate the solution algorithm used in pencil-and-paper calculations (i.e., processing each column of numbers sequentially), which may invoke more visual working memory resources than phonological working memory resources (see also Heathcote, 1994). Horizontally presented problems, in contrast, activate decomposition strategies (e.g., solving $45+9$ as $45+$ $5+4$ ), which may be more likely to involve verbal codes, and thus involve phonological working memory resources (see also Shanahan, 2009).

In sum, Trbovich and LeFevre's (2003) results indicate that visual codes are activated most strongly for problems in vertical format; in the present study, we tested whether this would be true for operations other than addition. Consistent with Trbovich and LeFevre's overall results, we predicted that both subtraction and multiplication would involve visual working memory. Complex arithmetic may routinely require visual working memory when the problems are displayed visually (vs. presented auditorily; Deslauriers, Ouellette, Barnes, \& LeFevre, 2008; cf. Noël, Désert, Aubrun, \& Seron, 2001). Subtraction may further invoke visual working memory processes, because it is likely to activate an internal magnitude code. In agreement with Trbovich and LeFevre's format effects, we also predicted larger visual load effects for problems vertically presented than for those presented horizontally. The inclusion of separate groups of participants educated in Canada and China allowed us to test whether the interaction between working memory component (phonological vs. visuospatial) and presentation format (horizontal vs. vertical) is universal across cultures.

The involvement of working memory may also depend on problem complexity, which, in complex arithmetic, is based generally on the number or difficulty of necessary carry or borrow operations. Carrying is needed when the 
sum or the product of the units is larger than 10 , which means that an amount must be carried from the units to the tens (e.g., $56+28,6 \times 24)$. Borrowing is needed when there is a shortfall (e.g., $52-17$ ), resulting in a borrow operation from the tens to the units. Although evidence for the role of the central executive in carrying and borrowing is very strong (Ashcraft \& Kirk, 2001; Fürst \& Hitch, 2000; Imbo, Vandierendonck, \& De Rammelaere, 2007; Imbo, Vandierendonck, \& Vergauwe, 2007; Seitz \& Schumann-Hengsteler, 2002), the involvement of phonological and visuospatial working memory resources in these processes is still debated. Some researchers did observe significant phonological working memory involvement in carrying and borrowing for Dutch- and German-educated adults (e.g., Imbo, Vandierendonck, \& De Rammelaere, 2007; Imbo, Vandierendonck, \& Vergauwe, 2007; Seitz \& Schumann-Hengsteler, 2000, 2002), whereas others did not observe such effects in Britishand Canadian-educated adults (e.g., Fürst \& Hitch, 2000; Logie, Gilhooly, \& Wynn, 1994; Trbovich \& LeFevre, 2003). Evidence for a role of the visuospatial sketchpad in carrying or borrowing has not been found in multidigit arithmetic for British-, French-, or Canadian-educated adults (Logie et al., 1994; Noël et al., 2001; Trbovich \& LeFevre, 2003).

In summary, in the present research, we investigated the role of phonological and visual working memory as a function of presentation format (horizontal vs. vertical), problem complexity (easy vs. difficult), and operation (subtraction vs. multiplication). We hypothesized that both subtraction and multiplication would be affected by a phonological working memory load, because both operations involve retention of intermediate solutions (Noël et al., 2001; Trbovich \& LeFevre, 2003). Similarly, we expected that both subtraction and multiplication would be affected by a visual memory load (Deslauriers et al., 2008; Trbovich \& LeFevre, 2003) but that the effect may be greater for subtraction, on the view that solution of simple subtraction problems involves internal magnitude or number line representations (Lee \& Kang, 2002). We also hypothesized that the decrements in performance would be greater for horizontally presented problems under a phonological load and for vertically presented problems under a visual load (Trbovich \& LeFevre, 2003), to the extent that these orientations enhance or exacerbate the activation of the respective working memory loads.

Last but not least, the inclusion of Chinese- and Canadian-educated participants allowed us to test the extent to which these results are generalizable across culture. Indeed, the respective interactions between working memory load and presentation format (Trbovich \& LeFevre, 2003) and between working memory load and operation (Lee \& Kang, 2002) have never been tested across cultures; it is thus perfectly possible that they are not universal. We expected the Chinese-educated participants to be more skilled than the Canadian-educated participants at arithmetic and thus to show smaller effects of problem complexity. Chinese participants were also expected to show additional effects of phonological load on multiplication problems, because such problems rely heavily on rote verbal memory to access component facts (LeFevre \& Liu, 1997). However, we had no reason to predict differential patterns of working memory loads for subtraction problems across cultures.

\section{EXPERIMENT 1}

\section{Method}

\section{Participants}

We recruited 65 participants: 29 (14 men, 15 women; mean age 21 years) were native English-speaking university students who had received their education in Canada, and 36 (14 men, 22 women; mean age 24 years) were native Chinese-speaking students who had received their education in China but were currently residing and studying in Canada. Their first language was Chinese, and their second language was English. Everyone responded in English. ${ }^{1}$ Thirtynine individuals participated to fulfill a course requirement, and 26 were paid 12 Canadian dollars.

\section{Materials}

Subtraction task. Three sets of 20 subtraction problems were constructed, resulting in a total of 60 different problems. Each set was presented twice to each participant - that is, once in horizontal presentation and once in vertical presentation. The order of the three sets and the combination of the set with the working memory load conditions were counterbalanced. All problems consisted of two two-digit numbers that had two-digit answers (e.g., $85-27$ ). Two types of problems were excluded: (1) problems involving a 0 in the units (e.g., $30-12 ; 32-10$ ) or in the remainder (e.g., 46 - 26), and (2) problems involving a 9 in the units (e.g., $69-$ 25 or $73-29$ ) or in the remainder (e.g., $56-27$ ). In order to exclude answer-size effects, the correct remainders of each set were distributed equally among the $30 \mathrm{~s}, 40 \mathrm{~s}, 50 \mathrm{~s}, 60 \mathrm{~s}$, and 70 s (i.e., four problems per type). Half of the problems involved a borrow operation from the tens to the units (e.g., $61-18$; hard problems); the other half did not (e.g., $74-32$; easy problems). The size of the remainder was equal for no-borrow problems and one-borrow problems. We also controlled for the even/uneven status of the correct remainder (i.e., half of the remainders were even; the other half were uneven).

Phonological secondary task. In this task, the experimenter read letter strings of four consonants (e.g., TKXL) aloud. The participant had to retain these letters and repeat them aloud after three subtraction problems. ${ }^{2}$ After the participant's response, the experimenter presented a new four-letter string. For each correct letter that was remembered in its correct position, participants gained 1 point. The maximum score on each trial was 4 points (i.e., $100 \%$ correct). This task has been used previously in research on mental arithmetic (e.g., Imbo \& Vandierendonck, 2007a, 2007b), in which it has been shown that retaining letters interferes only with phonologically based calculation strategies (such as transformation and counting) and not with direct memory retrieval. The letter task was also tested individually (i.e., without the concurrent solving of arithmetic problems) for $2 \mathrm{~min}$. In this secondary-task-only condition, a 15 -sec interval was used between the presentation of the four-letter string and the question to repeat the letters.

Visual secondary task. The visual memory load consisted of a pattern of four asterisks arranged in a $4 \times 4$ array. A list with 20 different patterns of four asterisks each was constructed. To discourage the participants from using a verbal label as a memory aid, none of the asterisk patterns formed recognizable lines, figures, objects, or letters. On each trial, a pattern was selected randomly and presented on the screen. The participant had to retain the pattern and reproduce it after three subtraction problems. For reproduction, the participant had to click with the mouse in the four appropriate grid locations (indicated by squares). Following the response of the participant, a new four-asterisk pattern was presented. For each star that was reproduced on its correct position, participants gained 1 point. The maximum 
score on each trial was 4 points (i.e., $100 \%$ correct). This task has been used previously in research on mental arithmetic (e.g., Trbovich \& LeFevre, 2003) and word naming (e.g., Herdman \& Beckett, 1996), and it has been shown to require the maintenance of visual codes and to be relatively free of phonological operations (Hellige, Cox, \& Litvak, 1979). The star task was also performed alone (i.e., without the concurrent solving of arithmetic problems) for $2 \mathrm{~min}$. The secondary-task-only execution of the phonological task and the visual task took place just before the execution of the primary task in combination with the respective secondary task. This ordering permitted the participants to get used to the secondary-task execution.

French Kit. After the complex-arithmetic experiment, all participants completed a portion of a paper-and-pencil test of arithmetic skill, the French Kit (French, Ekstrom, \& Price, 1963). The test consisted of two subtests, one page with complex addition problems and one page with complex subtraction and multiplication problems. Participants were given $2 \mathrm{~min} /$ page and were instructed to solve the problems as fast and accurately as possible. The correct answers on both subtests were summed to yield a total score of arithmetic skill.

\section{Procedure}

Each participant was tested individually by the same experimenter. The experiment took place in a quiet room and lasted for approximately 1 hour. All participants solved 20 subtraction problems in each of the six conditions defined by the 2 (presentation format: horizontal, vertical) $\times 3$ (working memory load: no load, phonological, visual) design. For half of the participants, the experiment started with the horizontal presentation format; for the other half, the experiment started with the vertical presentation format. The order of the working memory loads was randomized across participants.

A trial started with a fixation point for $500 \mathrm{msec}$, after which the subtraction problem was presented in the center of the screen. The problem remained on screen until the participant responded. Timing began when the stimulus appeared and ended when the response triggered the sound-activated relay. To enable this sound-activated relay, participants wore a microphone that was activated when they spoke their answer aloud. This microphone was connected to a software clock accurate to $1 \mathrm{msec}$. On each trial, on-screen feedback was presented to the participants: a green "Correct" when their answer was correct and a red "Incorrect" when it was not.

\section{Results}

In total, $2.8 \%$ of the trials were spoiled due to failures of the sound-activated relay. All data were analyzed on the basis of the multivariate general linear model; and all reported results were significant at $p<.05$, unless mentioned otherwise. Two Canadian participants did not solve any borrow problems correctly and were removed from all further analyses. Thus, the final sample included $27 \mathrm{Ca}-$ nadian and 36 Chinese participants. An analysis of French Kit scores confirmed that the Chinese had a substantially higher level of arithmetic skill than did the Canadians [54 vs. $\left.34 ; F(1,61)=38.04, M S_{\mathrm{e}}=166, \eta_{\mathrm{p}}^{2}=.38\right]$.

\section{Secondary Task Performance}

Percentage correct on each secondary task was analyzed separately in an ANOVA with culture (Canadian vs. Chinese) as the between-subjects factor and repeated measures on condition (single, dual horizontal, dual vertical; see Table 1).

Phonological memory. Accuracy varied with condition $\left[F(2,60)=40.62, M S_{\mathrm{e}}=130, \eta_{\mathrm{p}}^{2}=.40\right]$. Participants were more accurate in the single-task condition (79\%) than in the dual-horizontal $(64 \%)$ or dual-vertical $(63 \%)$ condition $[F(1,61)=56.57$ and 65.98 , respectively]. Accuracies on the phonological task did not differ across dual-horizontal and dual-vertical conditions $(F<1)$. No other effects were significant $\left(F_{\mathbf{S}}<1\right)$.

Visual memory. Accuracy varied with condition $\left[F(2,60)=148.55, M S_{\mathrm{e}}=126, \eta_{\mathrm{p}}^{2}=.71\right]$. Participants were more accurate in the single-task condition ( $97 \%$ correct) than in the dual-horizontal condition $[74 \% ; F(1,61)=$ $141.63]$ and tended to be more accurate in the latter condition than in the dual-vertical condition $[70 \%$ correct; $F(1,61)=3.45, p=.06]$. Further planned comparisons showed that Canadian participants' accuracies did not differ across dual-horizontal and dual-vertical conditions ( $71 \%$ vs. $72 \% ; F<1)$. However, Chinese were significantly less accurate in the dual-vertical (69\% correct) than in the dual-horizontal condition $[76 \%$ correct; $F(1,61)=$ 5.95], which indicates that the Chinese need more visual working memory resources to solve vertically presented subtraction problems than to solve horizontally presented subtraction problems. This result extends the conclusion of Trbovich and LeFevre (2003), who observed that visual working memory resources were used more for vertically than for horizontally presented addition problems.

\section{Subtraction}

Latencies. Latencies on correctly solved problems were analyzed in a 2 (culture: Canadian vs. Chinese) $\times 2$ (presentation format: horizontal vs. vertical) $\times 3$ (working memory load: control vs. phonological vs. visual) $\times$ 2 (problem complexity: easy vs. difficult) ANOVA with repeated measures on the last three factors (see Table 2). Vertically presented problems were solved more quickly than were horizontally presented problems [ $3.6 \mathrm{vs} .4 .0 \mathrm{sec}$; $\left.F(1,61)=27.42, M S_{\mathrm{e}}=909,675, \eta_{\mathrm{p}}^{2}=.31\right]$ and easy problems were solved more quickly than were hard problems $\left[2.9\right.$ vs. $4.7 \mathrm{sec} ; F(1,61)=115.82, M S_{\mathrm{e}}=5,477,819, \eta_{\mathrm{p}}^{2}=$ .66]. The main effect of working memory load $[F(2,60)=$ $\left.12.13, M S_{\mathrm{e}}=966,070, \eta_{\mathrm{p}}^{2}=.17\right]$ showed that people were

Table 1

Mean Accuracies and Standard Errors on the Phonological and Visual Secondary Tasks

\begin{tabular}{|c|c|c|c|c|c|c|c|c|c|}
\hline \multirow[b]{3}{*}{ Task } & \multirow[b]{3}{*}{ Load Type } & \multicolumn{4}{|c|}{ Subtraction (Experiment 1) } & \multicolumn{4}{|c|}{ Multiplication (Experiment 2) } \\
\hline & & \multicolumn{2}{|c|}{ Canadian } & \multicolumn{2}{|c|}{ Chinese } & \multicolumn{2}{|c|}{ Canadian } & \multicolumn{2}{|c|}{ Chinese } \\
\hline & & $M$ & $S E$ & $M$ & $\overline{S E}$ & $M$ & $S E$ & $M$ & $S E$ \\
\hline \multirow[t]{3}{*}{ Phonological } & Single & 79.2 & 0.6 & 79.6 & 0.5 & 80.3 & 0.2 & 80.3 & 0.3 \\
\hline & Dual horizontal & 65.2 & 3.1 & 62.9 & 2.7 & 63.9 & 2.9 & 59.8 & 2.6 \\
\hline & Dual vertical & 64.1 & 3.0 & 61.8 & 2.6 & 64.6 & 2.9 & 62.6 & 2.6 \\
\hline \multirow[t]{3}{*}{ Visual } & Single & 97.0 & 0.8 & 96.0 & 0.6 & 95.4 & 1.2 & 96.5 & 0.7 \\
\hline & Dual horizontal & 70.2 & 3.0 & 76.0 & 2.5 & 76.0 & 2.9 & 78.8 & 2.7 \\
\hline & Dual vertical & 70.8 & 2.8 & 68.8 & 2.5 & 72.1 & 3.0 & 75.3 & 2.5 \\
\hline
\end{tabular}


Table 2

Mean Response Times (in Seconds) As a Function of Culture, Presentation Format, Condition, and Problem Complexity

\begin{tabular}{|c|c|c|c|c|c|c|c|c|c|c|c|c|c|c|c|c|c|}
\hline \multirow[b]{4}{*}{ Task } & \multirow[b]{4}{*}{ Load Type } & \multicolumn{8}{|c|}{ Subtraction (Experiment 1) } & \multicolumn{8}{|c|}{ Multiplication (Experiment 2) } \\
\hline & & \multicolumn{4}{|c|}{ Canadian } & \multicolumn{4}{|c|}{ Chinese } & \multicolumn{4}{|c|}{ Canadian } & \multicolumn{4}{|c|}{ Chinese } \\
\hline & & \multicolumn{2}{|c|}{ Easy } & \multicolumn{2}{|c|}{ Difficult } & \multicolumn{2}{|c|}{ Easy } & \multicolumn{2}{|c|}{ Difficult } & \multicolumn{2}{|c|}{ Easy } & \multicolumn{2}{|c|}{ Difficult } & \multicolumn{2}{|c|}{ Easy } & \multicolumn{2}{|c|}{ Difficult } \\
\hline & & $M$ & $S E$ & $M$ & $S E$ & $M$ & $S E$ & $M$ & $S E$ & $M$ & $S E$ & $M$ & $S E$ & $M$ & $S E$ & $M$ & $S E$ \\
\hline \multirow[t]{3}{*}{ Horizontal } & None & 3.44 & 0.3 & 5.89 & 0.5 & 2.15 & 0.1 & 3.44 & 0.1 & 3.89 & 0.3 & 5.19 & 0.5 & 2.40 & 0.1 & 3.05 & 0.2 \\
\hline & Phonological & 4.01 & 0.4 & 5.97 & 0.6 & 2.52 & 0.1 & 3.85 & 0.2 & 4.40 & 0.3 & 6.09 & 0.6 & 2.77 & 0.2 & 3.34 & 0.2 \\
\hline & Visual & 3.69 & 0.3 & 6.20 & 0.5 & 2.48 & 0.1 & 3.91 & 0.2 & 4.60 & 0.4 & 5.88 & 0.5 & 2.73 & 0.1 & 3.48 & 0.3 \\
\hline \multirow[t]{3}{*}{ Vertical } & None & 2.93 & 0.2 & 5.22 & 0.5 & 1.94 & 0.1 & 3.18 & 0.2 & 3.66 & 0.3 & 4.84 & 0.4 & 2.22 & 0.1 & 2.66 & 0.2 \\
\hline & Phonological & 3.24 & 0.4 & 6.07 & 0.6 & 2.35 & 0.1 & 3.55 & 0.3 & 4.32 & 0.3 & 5.60 & 0.5 & 2.70 & 0.2 & 3.25 & 0.2 \\
\hline & Visual & 3.26 & 0.3 & 5.80 & 0.5 & 2.23 & 0.1 & 3.37 & 0.2 & 4.23 & 0.3 & 5.36 & 0.4 & 2.67 & 0.2 & 3.27 & 0.2 \\
\hline
\end{tabular}

faster in the no-load condition $(3.5 \mathrm{sec})$ than under either a phonological load $[3.9 \mathrm{sec} ; F(1,61)=17.11]$ or a visual load $[3.9 \mathrm{sec} ; F(1,61)=21.12]$. They were equally slow in both working memory load conditions $(F<1)$. As expected, Chinese $(2.9 \mathrm{sec})$ were significantly faster than Canadian participants $\left[4.6 \mathrm{sec} ; F(1,61)=24.29, M S_{\mathrm{e}}=\right.$ $\left.22,817,190, \eta_{\mathrm{p}}^{2}=.28\right]$. Culture further interacted with problem complexity $\left[F(1,61)=11.39, M S_{\mathrm{e}}=5,477,819\right.$, $\left.\eta_{\mathrm{p}}^{2}=.16\right]$. The problem complexity effect was larger for Canadian $(2.4 \mathrm{sec})$ than for Chinese $(1.3 \mathrm{sec})$ participants. The culture $\times$ presentation format and culture $\times$ working memory load interactions did not reach significance (both $p \mathrm{~s}>.25)$.

Errors. Percentages of errors were analyzed in a similar $2 \times 2 \times 3 \times 2$ ANOVA (see Table 3 ). The main effects of problem complexity $\left[F(1,60)=50.25, M S_{\mathrm{e}}=\right.$ $\left.410, \eta_{\mathrm{p}}^{2}=.46\right]$ and culture $\left[F(1,60)=6.14, M S_{\mathrm{e}}=561\right.$, $\left.\eta_{\mathrm{p}}^{2}=.09\right]$ were significant. Error rates were higher on difficult problems $(16.7 \%)$ than on easy problems $(6.0 \%)$ and higher for Canadian (13.5\%) than for Chinese $(9.2 \%)$ participants. Culture and problem complexity also interacted $\left[F(1,60)=5.09, M S_{\mathrm{e}}=410, \eta_{\mathrm{p}}^{2}=.08\right]$. The problem complexity effect was larger for Canadian (14.1\%) than for Chinese (7.3\%) participants. The culture $\times$ presentation format and culture $\times$ working memory load interactions did not reach significance (both $p$ s $>.55$ ).

\section{Discussion}

The results of the present experiment showed that people use both phonological and visual working memory resources when solving multidigit subtraction problems. Both Canadian and Chinese participants showed signifi- cant decrements in solution times in the dual-task conditions. In accord with previous findings about cultural differences, Chinese participants performed better on the subtraction problems than did the Canadian participants: They solved the problems more quickly and more accurately and were less affected by problem complexity (Campbell \& Xue, 2001; Imbo \& LeFevre, 2009). However, only the Chinese participants showed differential decrements related to presentation format (cf. Trbovich $\&$ LeFevre, 2003). They made more errors on the visual secondary task when solving vertically presented problems than when solving horizontally presented problems. This result shows that Trbovich and LeFevre's results may not be generalizable across operations or across cultural groups.

Experiment 2 was similar to Experiment 1, except that multiplication problems were tested instead of subtraction problems. The predictions for multiplication were somewhat different than those for subtraction. In particular, Lee and Kang (2002) reported that visuospatial working memory resources were important for subtraction (as was found in Experiment 1), whereas phonological resources were important for multiplication. Thus, we might expect multiplication to be more affected by phonological loads than by visual loads. Results for non-Asian groups have suggested that complex multiplications require phonological working memory and executive resources, but no studies (other than Lee \& Kang) have used visuospatial working memory loads with multiplication problems. Thus, the specificity of working memory components in relation to multiplication has not been tested across cultures for complex problems.

Table 3

Error Rates (\%) As a Function of Culture, Presentation Format, Condition, and Problem Complexity

\begin{tabular}{|c|c|c|c|c|c|c|c|c|c|c|c|c|c|c|c|c|c|}
\hline \multirow[b]{4}{*}{ Task } & \multirow[b]{4}{*}{ Load Type } & \multicolumn{8}{|c|}{ Subtraction (Experiment 1) } & \multicolumn{8}{|c|}{ Multiplication (Experiment 2) } \\
\hline & & \multicolumn{4}{|c|}{ Canadian } & \multicolumn{4}{|c|}{ Chinese } & \multicolumn{4}{|c|}{ Canadian } & \multicolumn{4}{|c|}{ Chinese } \\
\hline & & \multicolumn{2}{|c|}{ Easy } & \multicolumn{2}{|c|}{ Difficult } & \multicolumn{2}{|c|}{ Easy } & \multicolumn{2}{|c|}{ Difficult } & \multicolumn{2}{|c|}{ Easy } & \multicolumn{2}{|c|}{ Difficult } & \multicolumn{2}{|c|}{ Easy } & \multicolumn{2}{|c|}{ Difficult } \\
\hline & & $M$ & $S E$ & $M$ & $S E$ & $M$ & $S E$ & $M$ & $S E$ & $M$ & $S E$ & $M$ & $S E$ & $M$ & $S E$ & $M$ & $S E$ \\
\hline \multirow{3}{*}{ Horizontal } & None & 7.46 & 1.9 & 18.31 & 4.3 & 5.03 & 1.3 & 11.82 & 2.2 & 10.08 & 2.3 & 17.30 & 3.4 & 5.20 & 1.3 & 9.56 & 1.8 \\
\hline & Phonological & 5.69 & 1.6 & 22.42 & 3.7 & 5.5 & 1.2 & 13.07 & 2.1 & 10.93 & 1.7 & 15.62 & 2. & 7.33 & 1.4 & 14.09 & 2.1 \\
\hline & Visual & 8.96 & 2.3 & 20.19 & 4.4 & 7.43 & 1.5 & 11.11 & 2.1 & 9.51 & 2.2 & 18.50 & 3.2 & 3.80 & 0.8 & 11.77 & 1.9 \\
\hline \multirow[t]{3}{*}{ Vertical } & None & 4.69 & 1.3 & 19.54 & 4.1 & 5.59 & 1.5 & 13.70 & 2.8 & 7.78 & 1.8 & 18.45 & 3.8 & 4.81 & 1.1 & 6.95 & 1.7 \\
\hline & Phonological & 4.23 & 1.3 & 21.77 & 3.9 & 5.85 & 1.2 & 14.33 & 2.0 & 8.40 & 1.6 & 17.20 & 4.2 & 6.24 & 1.4 & 9.38 & 1.5 \\
\hline & Visual & 7.92 & 2.9 & 21.12 & 4.5 & 3.82 & 1.0 & 13.05 & 2.6 & 8.04 & 1.7 & 18.13 & 3.5 & 7.52 & 1.2 & 9.97 & 2.0 \\
\hline
\end{tabular}




\section{EXPERIMENT 2}

\section{Method}

\section{Participants}

We recruited 67 participants: 30 (16 men, 14 women; mean age 24 years) were native English-speaking university students who had received their education in Canada, and 37 (14 men, 23 women; mean age 23 years) were Chinese-speaking university students who had received their education in China but were currently living and studying in Canada. Their first language was Chinese, and their second language was English. Everyone responded in English. Fifty individuals participated for course credit, and 17 were paid $12 \mathrm{Ca}$ nadian dollars.

\section{Procedure and Tasks}

The set of 20 multiplication problems consisted of one-digit $X$ two-digit problems that met the following restrictions: (1) The twodigit number was not a decade (e.g., $3 \times 50),(2)$ the correct product was not a decade (e.g., $5 \times 16)$, and $(3)$ the one-digit number was not 1,2 , or 9 (e.g., $2 \times 14$ ). Further, all correct products were larger than 30 and smaller than 90 . In this set of 20 problems, there were 3 problems without a carry operation (e.g., $4 \times 12$ ), 9 problems with a carry value of 1 (e.g., $4 \times 14), 6$ problems with a carry value of 2 (e.g., $4 \times 16$ ), and 2 problems with a carry value of 3 (e.g., $4 \times 18$ ). Problems with a carry value of 0 or 1 were coded as easy; problems with a carry value of 2 or 3 were coded as difficult. The same set of problems was presented six times to each participant. The procedure, the secondary tasks, and the French Kit were identical to those in the previous experiments.

\section{Results}

In total, $2.8 \%$ of the trials were spoiled due to failures of the sound-activated relay. All data were analyzed on the basis of the multivariate general linear model, and all reported results were significant at $p<.05$, unless otherwise indicated. An analysis of French Kit scores confirmed that the Chinese participants had a higher level of arithmetic skill than the Canadian participants [ 48 vs. 30 ; $\left.F(1,65)=38.25, M S_{\mathrm{e}}=139, \eta_{\mathrm{p}}^{2}=.37\right]$.

\section{Secondary Task Performance}

Percentage correct on each secondary task was analyzed separately in an ANOVA with culture (Canadian vs. Chinese) as a between-subjects factor and repeated measures on condition (single, dual-horizontal, dual-vertical; see Table 1).

Phonological memory. The main effect of condition was significant $\left[F(2,64)=70.46, M S_{\mathrm{e}}=151, \eta_{\mathrm{p}}^{2}=.52\right]$. Participants were more accurate in the single-task condition $(80 \%$ correct) than in the dual-horizontal $(62 \%$ correct) and the dual-vertical (64\% correct) condition $[F(1,65)=90.00$ and 76.78 , respectively $]$. Accuracies on the phonological task did not differ across dual-horizontal and dual-vertical conditions $(F<1)$. The main effect of culture and the interaction between culture and condition did not reach statistical significance (both $F \mathrm{~s}<1$ ).

Visual memory. A similar ANOVA was conducted on the accuracies of the visual secondary task (see Table 1). The main effect of condition was significant $[F(2,64)=$ $\left.91.14, M S_{\mathrm{e}}=143, \eta_{\mathrm{p}}^{2}=.59\right]$. Participants were more accurate in the single-task condition ( $96 \%$ correct) than in the dual-horizontal (77\% correct) and the dual-vertical $(74 \%$ correct) condition $[F(1,65)=88.37$ and 140.90 , respec- tively]. Accuracies on the visual task did not differ across dual-horizontal and dual-vertical conditions $[F(1,65)=$ $2.38, p=.13]$. The main effect of culture and the interaction between culture and condition did not reach statistical significance (both $F_{\mathrm{S}}<1$ ).

\section{Multiplication}

Latencies. Latencies on correctly solved problems were analyzed in a 2 (culture: Canadian vs. Chinese) $\times 2$ (presentation format: horizontal vs. vertical) $\times 3$ (working memory load: control vs. phonological vs. visual) $\times 2$ (problem complexity: easy vs. difficult) ANOVA with repeated measures on the last three factors (see Table 2). Participants responded faster on vertically presented problems than on horizontally presented problems [3.7 vs. $\left.4.0 \mathrm{sec} ; F(1,65)=8.46, M S_{\mathrm{e}}=1,500,118, \eta_{\mathrm{p}}^{2}=.12\right]$ and faster on easy $(4.2 \mathrm{sec})$ than on difficult $(5.5 \mathrm{sec})$ problems $\left[F(1,65)=88.45, M S_{\mathrm{e}}=2,028,826, \eta_{\mathrm{p}}^{2}=.58\right]$. The main effect of working memory load $[F(2,64)=$ $\left.36.37, M S_{\mathrm{e}}=710,494, \eta_{\mathrm{p}}^{2}=.36\right]$ indicated that people were faster under no-load conditions $(3.5 \mathrm{sec})$ than under phonological $(4.1 \mathrm{sec})$ or under visual $(4.0 \mathrm{sec})$ load $[F(1,65)=48.81$ and 65.36 , respectively $]$. They were as slow under phonological load as under visual load $(F<1)$. Chinese $(2.9 \mathrm{sec})$ responded faster than Canadian $(4.8 \mathrm{sec})$ participants did $\left[F(1,65)=26.63, M S_{\mathrm{e}}=\right.$ $\left.28,673,880, \eta_{\mathrm{p}}^{2}=.29\right]$. Culture further interacted with problem complexity $\left[F(1,65)=12.75, M S_{\mathrm{e}}=2,028,826\right.$, $\left.\eta_{\mathrm{p}}^{2}=.16\right]$. The problem complexity effect was larger in Canadian $(1.3 \mathrm{sec})$ than in Chinese $(0.6 \mathrm{sec})$ participants. The culture $\times$ presentation format and culture $\times$ working memory load interactions did not reach significance (both $p s>.20$ ).

Errors. Percentages of errors were analyzed in a similar $2 \times 2 \times 3 \times 2$ ANOVA (see Table 3 ). The main effects of problem complexity and culture were significant $\left[F(1,65)=28.01, M S_{\mathrm{e}}=282, \eta_{\mathrm{p}}^{2}=.30\right.$, and $F(1,65)=$ $\left.9.96, M S_{\mathrm{e}}=555, \eta_{\mathrm{p}}^{2}=.13\right]$. Error rates were larger for difficult problems (13.8\%) than for easy problems (7.4\%) and larger for Canadian (13.2\%) than for Chinese $(8.0 \%)$ participants. The interaction across culture, problem complexity, and presentation format was significant $\left[F(1,65)=4.59, M S_{\mathrm{e}}=127, \eta_{\mathrm{p}}^{2}=.07\right]$. The problem complexity effect was larger for Canadian than for Chinese participants for vertically presented problems $[9.7 \%$ vs. $2.4 \% ; F(1,65)=7.01]$, but not for horizontally presented problems $(6.8 \%$ vs. $6.2 \% ; F<1)$. The interaction between presentation format and working memory load was tested separately for each culture to evaluate the prediction that the Chinese participants would be more affected by a phonological working memory load than by a visual load. As expected, no differential load effects were found for the Canadian participants. For the Chinese participants, however, the interaction was significant $\left[F(1,36)=4.67, \eta_{\mathrm{p}}^{2}=.11\right]$. For the Chinese participants, phonological load effects were significant for horizontally presented problems $[3.4 \% ; F(1,36)=$ $7.12]$, but not for vertically presented problems $(1.9 \%$; $p=.15)$. Visual load effects, in contrast, tended to be 
significant for vertically presented problems $[2.9 \%$; $F(1,36)=3.26, p=.07]$, but were not significant for horizontally presented problems $(0.4 \% ; F<1]$.

\section{Discussion}

The results of the present experiment showed that people use both phonological and visual working memory resources when solving multidigit multiplication problems. Solution times on the multiplication problems and performance on the load tasks showed significant decrements in the dual-task conditions. The Chinese participants performed better on the multiplication problems than did the Canadian participants: They solved the problems more quickly and more accurately, and they were less affected by problem complexity. As in Experiment 1, only the Chinese participants showed differential decrements related to presentation format. Patterns were different across experiments, however. In Experiment 2, Chinese-educated participants made more errors on the multiplication task under a phonological load in horizontal than in vertical format, whereas in Experiment 1, subtraction was more affected by a visual load. Hence, the results of both experiments indicate that the interaction between working memory resources (phonological vs. visuospatial) and presentation format (horizontal vs. vertical) is not unconditionally valid. In the present research, interactions were found for Chinese but not for Canadian participants, in that Canadians showed decrements in performance for both operations, both presentation formats, and both types of working memory load. In the General Discussion, we interpret the present results in the light of previous studies (Lee \& Kang, 2002; Trbovich \& LeFevre, 2003) and explore the question of why results may not always be generalizable across arithmetic operations and cultural groups.

\section{GENERAL DISCUSSION}

We conducted two experiments to investigate cultural differences in complex arithmetic, as well as their interaction with presentation format, working memory load, and problem complexity. As predicted, both $\mathrm{Ca}$ nadian and Chinese participants relied on phonological and visual working memory resources when solving complex arithmetic problems. Decrements occurred for both groups in subtraction and in multiplication. Differential working memory load effects as a function of presentation format (cf. Trbovich \& LeFevre, 2003) and operation (cf. Lee \& Kang, 2002) were significant only for Chinese participants, however. Chinese participants showed greater phonological load effects on multiplication problems in horizontal than in vertical format, consistent with the view that the horizontal format exacerbated the deleterious effect of the phonological load. Chinese participants also showed greater decrements on the visual load task for vertically presented subtractions than for horizontally presented subtractions, supporting the view that the vertical format exacerbated the effect of the visual load. Canadians, in contrast, showed decre- ments to both types of loads in both format conditions across operations.

The observation of significant working memory load effects in the Canadian sample is in agreement with two earlier studies on Canadians' complex arithmetic performance: Imbo and LeFevre (2009) found phonological load effects, and Trbovich and LeFevre (2003) found both visual and phonological load effects. Both studies tested only complex addition, however, so the present study extends these results to other operations by showing that Canadians need both phonological and visual working memory resources when performing complex subtraction and multiplication. Given the higher level of mathematical skill in Asian than in Canadian participants (Campbell \& Xue, 2001; Imbo \& LeFevre, 2009; LeFevre \& Liu, 1997; Siegler \& Mu, 2008), we had anticipated that working memory load effects might be less in the Chinese than in the Canadian participants. However, Chinese-educated participants in the present research did slow down under phonological and visual working memory loads, even though other Chinese participants did not rely on executive working memory resources when solving complex addition problems (Imbo $\&$ LeFevre, 2009). Hence, Chinese may not rely on the controlling or monitoring function of their working memory when solving complex additions, but they do rely on the storage capacity of their working memory when solving complex subtractions and multiplications. In sum, both Chinese and Canadian participants relied on phonological and visual working memory when solving complex subtractions and multiplications, suggesting that they maintained intermediate solutions in a verbal format and on a mental blackboard. How the mental blackboard maintains visual information and which specific role it plays in mental arithmetic are questions that should be addressed in future research.

We also observed some evidence for the link between working memory and presentation format (cf. Trbovich \& LeFevre, 2003). Whereas Canadian participants did not show differential load effects as a function of presentation format, Chinese participants did: In the subtraction experiment, they relied on visual memory more for vertically than for horizontally presented problems, and in the multiplication experiment, they relied on phonological memory more for horizontally than for vertically presented problems. Trbovich and LeFevre found that addition problems in vertical format involved visual working memory to a greater degree, whereas those in horizontal format involved phonological working memory. Thus, although aspects of the present results are in agreement with those of Trbovich and LeFevre, the present results also show that one should be careful when generalizing effects of working memory load over operations or over cultural groups.

Differential load effects as a function of presentation format seem to be detected more easily in addition than in other, less practiced operations such as subtraction and multiplication. Obviously, performance measures such as response times and error rates are more variable for 
less practiced operations, and this variability may have obscured possible interactions. The variability is even greater in less skilled participants, such as the Canadians in our study. The performance of the highly skilled Chinese participants was more efficient (i.e., faster and less erroneous) and correspondingly less variable than that of the Canadian participants, which may have increased the chance of observing differential load effects for the former.

Another explanation for the fact that differential load effects were culturally specific may be found in the different educational approaches in Asian and non-Asian cultures. In the Chinese education system, the multiplication tables are taught by using rhyming formulae and involve extensive training in the form of drill and rote verbal memory (Zhang \& Zhou, 2003), accounting for the connection between the phonological loop and horizontally presented multiplication problems (cf. Experiment 2). Additions and subtractions, in contrast, are often taught by using the abacus (Cantlon \& Brannon, 2007; Stigler, 1984), which relies on a vertical mental representation requiring visual working memory resources (cf. Experiment 1). Imaging studies indeed showed that the mental abacus operation is associated with enhanced activity in the brain areas relevant for visuospatial information processing (e.g., Hanakawa, Honda, Okada, Fukuyama, \& Shibasaki, 2003; Wu et al., 2009). Hence, the distinct Chinese training regimens may lead to distinct mental processes and representations across operations.

Different educational approaches are more common in North America than in China. First of all, North Americans are less thoroughly drilled and trained than are Asians. This lack of automaticity implies a greater use of executive working memory resources in North Americans than in Asians (cf. Imbo \& LeFevre, 2009). Further, the more exploratory approach to learning arithmetic favored in North American education may result in a greater variety in strategy use (cf. Campbell \& Xue, 2001; LeFevre \& Liu, 1997). Canadian participants' highly variable strategy use may increase the need for phonological and visual working memory resources, if the information has to be stored (as intermediate solutions) differentially across problems and operations. Hence, the specific functions of the phonological loop and the visual sketchpad may be more consistent for Chinese than for Canadian participants.

Also note that the differential load effects were found only in errors (i.e., secondary task errors in Experiment 1 and multiplication errors in Experiment 2). Similarly, in Trbovich and LeFevre (2003), differential effects of working memory load were found only on error scores (combined across the secondary and arithmetic tasks). These patterns suggest that the role of working memory subsystems in complex arithmetic is connected more strongly to maintaining and accessing representations than to the speed with which the processing is implemented. This observation suggests that further research would be fruitful in exploring in more detail how working memory is involved in the problem-solving process.
Finally, we observed cultural differences in the problem complexity effect as well. The Chinese participants were more proficient in solving carry and borrow problems than were the Canadian participants. Cultural differences in problem solving have been shown in the domain of simple arithmetic (e.g., Campbell \& Xue, 2001; LeFevre \& Liu, 1997) and, for one study, in complex arithmetic (Imbo \& LeFevre, 2009). Speed and accuracy scores are lower for Canadian than for Chinese participants - and these differences increase as problem complexity increases for both simple arithmetic (Campbell \& Xue, 2001; LeFevre \& Liu, 1997) and complex arithmetic (the present study; see also Imbo \& LeFevre, 2009). Hence, the Chinese are not only generally more efficient, they are also specifically more efficient on the difficult carry and borrow problems, suggesting higher levels of competency in both declarative and procedural knowledge.

Despite the clear effect of problem complexity in both experiments, borrowing and carrying did not directly tax phonological or visual working memory. Research on the possible role of phonological working memory resources in carrying and borrowing has yielded equivocal results. Some researchers did observe significant phonological load effects that varied with problem complexity (e.g., Imbo, Vandierendonck, \& De Rammelaere, 2007; Imbo, Vandierendonck, \& Vergauwe, 2007; Seitz \& Schumann-Hengsteler, 2000, 2002), whereas others did not (e.g., Fürst \& Hitch, 2000; Logie et al., 1994; Trbovich \& LeFevre, 2003). One intriguing possibility is that the language of the participants may influence whether phonological resources are implicated in arithmetic solutions. The only studies in which phonological load effects on carrying and borrowing have been observed were conducted on Dutch-speaking (Imbo, Vandierendonck, \& De Rammelaere, 2007; Imbo, Vandierendonck, \& Vergauwe, 2007) or German-speaking (Seitz \& Schumann-Hengsteler, 2000, 2002) participants. The number language of these Germanic languages is different from those of English or Asian languages (i.e., 45 is pronounced as "five and forty"). This inverse order of pronunciation (relative to the Arabic digit form) may require extra phonological working memory involvement (see Brysbaert, Fias, \& Noël, 1998) - a hypothesis that needs to be tested in further studies. The absence of visual load effects on carrying or borrowing is in agreement with the very few studies on this topic (Logie et al., 1994; Noël et al., 2001; Trbovich \& LeFevre, 2003) and indicates that the visuospatial sketchpad is not involved in carrying or borrowing.

In summary, both Canadian and Chinese participants relied on phonological and visual working memory resources when solving complex subtraction and multiplication problems. Although the Chinese participants were more efficient than the Canadian participants were, the involvement of phonological and visual working memory recourses was comparable. However, differential working memory load effects were obtained only for Chinese and not for Canadian participants. More spe- 
cifically, Chinese showed a greater role for the phonological loop in horizontally presented than in vertically presented multiplication problems (Experiment 2) and a greater role for the visuospatial sketchpad in vertically than in horizontally presented subtraction problems (Experiment 1). Conclusions from earlier experiments conducted within a single culture thus cannot necessarily be generalized. Intercultural studies have a double advantage: They test for differences between cultures; and they test whether widely accepted conclusions can be generalized over cultures. Thus, more systematic comparisons of performance across different cultural groups and different arithmetic operations are likely to yield useful information for theories of how numerical information is processed and represented.

\section{AUTHOR NOTE}

Support for this research was provided by the Research Foundation Flanders (FWO Flanders) with a postdoctoral fellowship to I.I. and by the Natural Sciences and Engineering Research Council of Canada through a Discovery Grant to J.-A.L. Address correspondence to I. Imbo, Department of Experimental Psychology, Ghent University, Henri Dunantlaan 2, B-9000 Ghent, Belgium (e-mail: ineke.imbo@ugent.be).

\section{REFERENCES}

AshCRAFT, M. H. (1995). Cognitive psychology and simple arithmetic: A review and summary of new directions. Mathematical Cognition, 1, 3-34.

AsHCRAFT, M. H., \& KIRK, E. P. (2001). The relationships among working memory, math anxiety, and performance. Journal of Experimental Psychology: General, 130, 224-237.

BADDELEY, A. D. (2000). The episodic buffer: A new component of working memory? Trends in Cognitive Sciences, 4, 417-423.

Baddeley, A. D., \& Hitch, G. J. (1974). Working memory. In G. H. Bower (Ed.), The psychology of learning and motivation (Vol. 8, pp. 47-90). San Diego: Academic Press.

Brysbaert, M., Fias, W., \& NoËL, M. P. (1998). The Whorfian hypothesis and numerical cognition: Is "twenty-four" processed in the same way as "four-and-twenty"? Cognition, 66, 51-77.

CAmpbell, J. I. D., \& XUE, Q. (2001). Cognitive arithmetic across cultures. Journal of Experimental Psychology: General, 130, 299-315.

Cantlon, J. F., \& Brannon, E. M. (2007). Adding up the effects of cultural experience on the brain. Trends in Cognitive Sciences, 11, $1-4$.

Cohen, L., \& Dehaene, S. (2000). Calculating without reading: Unsuspected residual abilities in pure alexia. Cognitive Neuropsychology, 17, 563-583.

Cohen, L., Dehaene, S., Chochon, F., Lehéricy, S., \& Naccache, L. (2000). Language and calculation within the parietal lobe: A combined cognitive, anatomical and fMRI study. Neuropsychologia, 38, 1426-1440.

Dehaene, S. (1992). Varieties of numerical abilities. Cognition, 44, $1-42$.

Dehaene, S. (1997). The number sense: How the mind creates mathematics. New York: Oxford University Press.

Dehaene, S., \& CoHEN, L. (1995). Towards an anatomical and functional model of number processing. Mathematical Cognition, 1, 83-120.

Dehaene, S., \& Cohen, L. (1997). Cerebral pathways for calculation: Double dissociation between rote verbal and quantitative knowledge of arithmetic. Cortex, 33, 219-250.

Deslauriers, W. A., Ouellette, G. P., Barnes, M., \& LeFevre, J.-A (2008). To see or not to see: The visual component of complex mental arithmetic. In B. C. Love, K. McRae, \& V. M. Sloutsky (Eds.), Proceedings of the 30th Annual Conference of the Cognitive Science Society (pp. 241-246). Austin, TX: Cognitive Science Society.

DeStefano, D., \& LeFevre, J.-A. (2004). The role of working memory in mental arithmetic. European Journal of Cognitive Psychology, 16, 353-386.
French, J. W., Exstrom, R. B., \& Price, I. A. (1963). Kit of reference tests for cognitive factors. Princeton, NJ: Educational Testing Service.

FüRST, A. J., \& HiTch, G. J. (2000). Separate roles for executive and phonological components of working memory in mental arithmetic. Memory \& Cognition, 28, 774-782.

Hanakawa, T., Honda, M., Okada, T., Fukuyama, H., \& ShibaSAKI, H. (2003). Neural correlates underlying mental calculation in abacus experts: A functional magnetic resonance imaging study. NeuroImage, 19, 296-307.

Heathсоте, D. (1994). The role of visual-spatial working memory in the mental addition of multi-digit addends. Cahiers de Psychologie Cognitive, 13, 207-245.

Hellige, J. B., Cox, P. J., \& LitvaK, L. (1979). Information processing in the cerebral hemispheres: Selective hemispheric activation and capacity limitations. Journal of Experimental Psychology: General, 108, 251-279.

Herdman, C. M., \& Beckett, B. L. (1996). Code-specific processes in word naming: Evidence supporting a dual-route model of word recognition. Journal of Experimental Psychology: Human Perception \& Performance, 22, 1149-1165.

Imbo, I., \& LeFeVre, J.-A. (2009). Cultural differences in complex addition: Efficient Chinese versus adaptive Belgians and Canadians. Journal of Experimental Psychology: Learning, Memory, \& Cognition, 35, 1465-1476.

IMBo, I., \& VANDIERENDONCK, A. (2007a). Do multiplication and division strategies rely on executive and phonological working memory resources? Memory \& Cognition, 35, 1759-1771.

IMBO, I., \& VANDIERENDONCK, A. (2007b). The role of phonological and executive working memory resources in simple-arithmetic strategies. European Journal of Cognitive Psychology, 19, 910-933.

Imbo, I., Vandierendonck, A., \& De Rammelaere, S. (2007). The role of working memory in the carry operation of mental arithmetic: Number and value of the carry. Quarterly Journal of Experimental Psychology, 60, 708-731.

Imbo, I., Vandierendonck, A., \& Vergauwe, E. (2007). The role of working memory in carrying and borrowing. Psychological Research, 71, 467-483.

LeE, K.-M., \& KanG, S.-Y. (2002). Arithmetic operation and working memory: Differential suppression in dual tasks. Cognition, 83, B63B68.

LeFevre, J.-A., \& LiU, J. (1997). The role of experience in numerical skill: Multiplication performance in adults from China and Canada. Mathematical Cognition, 3, 31-62.

Logie, R. H., Gilhooly, K. J., \& WynN, V. (1994). Counting on working memory in arithmetic problem solving. Memory \& Cognition, 22, 395-410.

NoËL, M.-P., Désert, M., Aubrun, A., \& Seron, X. (2001). Involvement of short-term memory in complex mental calculation. Memory \& Cognition, 29, 34-42.

Seitz, K., \& Schumann-Hengsteler, R. (2000). Mental multiplication and working memory. European Journal of Cognitive Psychology, 12, 552-570.

Seitz, K., \& Schumann-Hengsteler, R. (2002). Phonological loop and central executive processes in mental addition and multiplication. Psychologische Beiträge, 44, 275-302.

SeYler, D. J., Kirk, E. P., \& Ashcraft, M. H. (2003). Elementary subtraction. Journal of Experimental Psychology: Learning, Memory, \& Cognition, 29, 1339-1352.

Shanahan, T. (2009). Strategy and procedures in adults' solution of multi-digit addition problems. Unpublished $\mathrm{PhD}$ thesis, Carleton University.

Siegler, R. S., \& Mu, Y. (2008). Chinese children excel on novel mathematics problems even before elementary school. Psychological Science, 19, 759-763.

STIGLER, J. W. (1984). "Mental abacus": The effect of abacus training on Chinese children's mental calculation. Cognitive Psychology, 16, 145-176.

Trbovich, P. L., \& LeFevre, J.-A. (2003). Phonological and visual working memory in mental addition. Memory \& Cognition, 31, 738-745.

Wu, T.-H., Chen, C.-L., Huang, Y.-H., Liu, R.-S., Hsieh, J. C., \& Lee, J. J. S. (2009). Effects of long-term practice and task complexity on brain activities when performing abacus-based mental calculations: 
A PET study. European Journal of Nuclear Medicine \& Molecular Imaging, 36, 436-445.

ZHANG, H., \& ZHOU, Y. (2003). The teaching of mathematics in Chinese elementary schools. International Journal of Psychology, 38, 286-298.

\section{NOTES}

1. Although having to respond in their second language might have been a small disadvantage for the Chinese, the results show that they nevertheless performed better than the Canadians did. Thus, although a possible effect of language of testing cannot be discounted in the present research, this issue seems unlikely to have compromised the main conclusions.

2 . In order to have seven series of 3 arithmetic problems each, 1 subtraction problem (Experiment 1) and 1 multiplication problem (Experiment 2) were repeated. This problem was chosen randomly from the set of 20 problems.

(Manuscript received April 6, 2009;

revision accepted for publication September 7, 2009.) 